\title{
On Self-Adjoint Partial Differential Equations of the Second Order.
}

By E. T. Copson.

Received 14th October 1924. Read 7th November 1924.

$\S 1$. Let $F(u)=\sum_{i, k} A_{i k} \frac{\partial^{2} u}{\partial x_{i} \partial x_{k}}+\sum_{i} B_{i} \frac{\partial u}{\partial x_{i}}+C u$ be a linear differential expression involving $n$ independent variables $x_{i}$, the coefficients $A_{i k}, B_{i}$ and $C$ being functions of the independent variables but not involving the dependent variable $u$. Associated with $F(u)$ is the adjoint expression

$$
G(v)=\sum_{i, k} \frac{\partial^{2}}{\partial x_{i} \partial x_{k}}\left(A_{i k} v\right)-\sum_{i} \frac{\partial}{\partial x_{i}}\left(B_{i} v\right)+C v^{*}
$$

If the expressions $F(u)$ and $G(u)$ are identical, $F(u)$ is said to be self-adjoint, and the equation $F(u)=0$ is a self-adjoint linear partial differential equation of the second order.

If a second order linear partial differential equation is obtained by annulling the variation of an integral according to the methods of the calculus of variations, this equation must be self-adjoint, and conversely. Also by applying the theory of continuous groups of transformations to such an integral, certain conservation theorems $\dagger$ satisfied by the solution of the partial differential equation will be obtained.

Consequently the investigation of the conditions which the coefficients of a general linear second order partial differential equation must satisfy to be self-adjoint appears to be of interest.

" See Courant u. Hilbert: Methoden der Mathematischen Physik. Band I., Kap. IV., \$8.

† E.g. in Dynamics, the theorems of conservation of energy and momen. tum. See a paper by Emay Noether: Gött. Nach. (1918), p. 238. Also the present author's paper Proc. Edin. Math. Soc., 42, p 61. 
The principal result here obtained is that in general a linear partial differential equation of the second order with constant coefficients can be made self-adjoint by multiplication by a factor $e_{i}^{\Sigma \lambda_{i} x_{i}}$ where the coefficients $\lambda_{i}$ are certain constants. The exceptional case is when the equation is of parabolic type.

\$2. Let us denote derivatives by suffixes. Then we may write $F(u)=\sum_{i, k} a^{i k} u_{i k}+2 \sum_{i} b^{i} u_{i}+c u$, where the coefficients $a^{i k}, b^{i}, c$ are functions of the variables $x_{i}$, and where $a^{i k}=a^{k i}$. We easily see that

$$
G(v)=\sum_{i, k} a^{i k} v_{i k}+2 \sum_{i} v_{i}\left(\sum_{k} a_{k}^{i k}-b^{i}\right)+c v+v \sum_{i}\left(\sum_{k} a_{i k}^{i k}-2 b_{i}^{i}\right) .
$$

If the coefficients satisfy the $n$ first order partial differential equations $\sum a_{k}^{i k}=2 b^{i} \quad(i=1,2, \ldots \ldots n)$ then $F(u)$ is identical with $G(u)$ and is consequently self-adjoint. If the coefficients do not satisfy these equations, it may be possible to find a function $\phi\left(x_{1}, x_{2}, \ldots \ldots x_{n}\right)$ which is such that $\phi F(u)$ is self-adjoint. For the purpose of finding conservation theorems, this would be just as useful. The function $\phi$ must satisfy the $n$ equations

$$
\sum_{i} \phi a_{k}^{i k}+\sum_{i} \phi_{k} a^{i k}=2 \phi b^{i} \quad(i=1,2, \ldots \ldots n) .
$$

The case of particular interest is the equation with constant coefficients. Such an equation can only be self-adjoint if the coefficients $b^{i}$ are all zero. But if $\phi F(u)$ is self-adjoint and the expression $F(u)$ has constant coefficients, $\phi$ must satisfy the $n$ equations $\sum_{k} a^{i k} \phi_{k}=2 l^{i} \phi \quad(i=1,2, \ldots \ldots n)$.

This system of equations has the solution $\phi=e^{\Sigma \lambda_{i} x_{i}}$ where

$$
2 b^{r}=a^{r 1} \lambda_{1}+a^{r 2} \lambda_{2}+\ldots \ldots+a^{r n} \lambda_{n}(r=1,2, \ldots n) .
$$

If we exclude the case of an equation of parabolic type which is such that the determinant of the coefticients $a^{i k}$ vanishes, the constants $\lambda_{i}$ can be uniquely determined; hence any second order linear non-parabolic partial differential equation with constant coefficients can be made self-adjoint by multiplication by a factor $\epsilon^{\Sigma \lambda_{i} x_{i}}$ and so can be derived from a calculus of variations problem. 
$\$ 3$. To discuss the case when the determinant $\left|a^{i k}\right|$ vanishes, it is convenient to make use of a linear change of the independent variables $x_{i}$ to $\underline{x}_{i}$, where $\underline{x}_{i}=\sum_{i} l_{k i} x_{k}$. Denoting $\frac{\partial u}{\partial x_{i}}$ by $\underline{u}_{i}$ and so on, we have

$$
F(u)=\sum_{i . k} u_{i k}\left(\sum a_{r, s}^{r k} l_{r i} l_{s k}\right)+2 \sum_{i} u_{i}\left(\sum_{i} b^{r} l_{r i}\right)+c u .
$$

Since $\left|a^{a}\right|=0$, we can choose $l_{11}, l_{21}, \ldots l_{n 1}$ so as to satisfy the $n$ equations $a^{1 s} l_{11}+a^{2 s} l_{21}+\ldots+a^{n s} l_{n 1}=0(s=1,2, \ldots n)$. When this is done, the expression $F(u)$ becomes

$$
\sum_{i, k}^{\prime} \underline{a}^{i k} \underline{u}_{i k}+2 \sum_{i}^{\prime} \underline{b}^{i} \underline{u_{i}}+c u+2 b^{i} \underline{u}
$$

where $\Sigma^{\prime}$ means that the summation is made over the values $2,3, \ldots n$. instead of $1,2, \ldots n$, and where the coefficients $a^{i k}, b^{i}$ are constants.

Now it can easily be shewn that $\phi F(u)$ will be self-adjoint after such a change only if it was before. The conditions then are that

$$
\begin{aligned}
2 b^{i} & =a^{i 2} \lambda_{2}+a^{i 3} \lambda_{3}+\ldots+a^{i n} \lambda_{n}(i=2,3, \ldots n) \\
2 b^{1} & =0
\end{aligned}
$$

where $\quad \phi=e_{i=}^{Y \lambda_{i}} \underline{X_{i}}$.

Hence if the expression $F(u)$ has constant coefficients which are such that $\left|a^{i k}\right|=0$, and if it becomes self-adjoint on multiplication by a factor $e^{\Sigma \lambda_{i} x_{i}}$, it must be reducible to an expression in $(n-1)$ independent variables.

Ex. 1. The equation $\frac{\hat{o}^{2} u}{\partial x^{2}}+\frac{\hat{\sigma}^{n} u}{\partial y^{2}}-\frac{1}{c^{2}} \frac{\hat{o}^{2} u}{\partial t^{2}}-\frac{\gamma}{c^{2}} \frac{\partial}{\partial t}-\lambda u=0$ is not self-adjoint. But considered in the form

$$
e^{\mathrm{\gamma} t}\left(\frac{\partial^{2} u}{\partial x^{2}}+\frac{\partial^{2} u}{\partial y^{2}}-\frac{1}{c^{2}} \frac{\partial^{2} u}{\partial t^{2}}-\frac{\gamma}{c^{2}} \frac{\partial u}{\partial t}-\lambda u\right)=0
$$


it is self-adjoint, and can be derived from the Calculus of Variations Problem

$$
\delta \iiint e^{r t}\left\{\left(\frac{\partial u}{d x}\right)^{2}+\left(\frac{\partial u}{d y}\right)^{2}-\frac{1}{c^{2}}\left(\frac{\partial u}{\partial t}\right)^{2}+\lambda u^{2}\right\} d x d y d t=0
$$

$E x$. 2. The equation $\frac{\partial^{2} u}{\partial x^{2}}+\frac{\partial^{2} u}{\partial y^{2}}+u \frac{\partial u}{\partial x}+\beta \frac{\partial u}{\partial y}+\gamma \frac{\partial u}{\partial z}+\lambda u=0$

is not self-adjoint, and can only be made self-adjoint by multiplication by an exponential factor when $\gamma=0$, that is, when the equation reduces to one in two independent variables. 